\title{
Metallicity at interphase boundaries due to polar catastrophe induced by charge density discontinuity
}

\author{
Arwa Albar ${ }^{1}$, Hassan Ali Tahini and Udo Schwingenschlögl \\ The electronic properties of interphase boundaries are of basic importance for most materials, particularly when those properties \\ deviate strongly from the bulk behavior. We introduce a mechanism that can result in metallicity at stoichiometric interphase \\ boundaries between semiconductors based on the idea of polar catastrophe, which is usually considered only in the context of \\ heterostructures. To this end, we perform $a b$ initio calculations within density functional theory to investigate the electronic \\ states at stoichiometric $\mathrm{SnO} \mathrm{SnO}_{2}(110)$ interphase boundaries. In this system, one would not expect polar catastrophe to have a \\ role according to state-of-the-art theory because the interface lacks formal charge discontinuity. However, we observe the \\ formation of a hole gas between the semiconductors $\mathrm{SnO}$ and $\mathrm{SnO}_{2}$. To explain these findings, we provide a generalized theory \\ based on the idea that the charge density discontinuity between $\mathrm{SnO}$ and $\mathrm{SnO}_{2}$, a consequence of lattice mismatch, drives a \\ polar catastrophe scenario. As a result, $\mathrm{SnO}_{/} \mathrm{SnO}_{2}(110)$ interphase boundaries can develop metallicity depending on the grain \\ size. The concept of metallicity due to polar catastrophe induced by charge density discontinuity is of general validity and \\ applies to many interphase boundaries with lattice mismatch.
}

NPG Asia Materials (2018) 10, e469; doi:10.1038/am.2017.236; published online 9 February 2018

\section{INTRODUCTION}

Tin oxide has two stoichiometric phases, the monoxide $(\mathrm{SnO})$ and the dioxide $\left(\mathrm{SnO}_{2}\right)$, the latter being frequently used in gas sensors, oxidation catalysts and solar cell electrodes, for example. ${ }^{1}$ Recently, $\mathrm{SnO}$ has garnered interest after a record hole mobility was reported in Caraveo-Frescas et al., ${ }^{2}$ which opens a multitude of possible applications. Although $\mathrm{SnO} / \mathrm{SnO}_{2}$ interphase boundaries form during the oxidation of $\mathrm{Sn}$, their properties are largely unknown, ${ }^{3}$ while in general oxide-oxide interfaces are receiving much attention due to a variety of exciting phenomena.,

An important example is the appearance of a quantum gas at the interface between the wide-band-gap semiconductors $\mathrm{LaAlO}_{3}$ and $\mathrm{SrTiO}_{3}{ }^{6}$ as well as at other $n$-type interfaces, ${ }^{7-11}$ whereas an insulating character has been reported for the p-type $\mathrm{LaAlO}_{3} / \mathrm{SrTiO}_{3},{ }^{6} \mathrm{LaVO}_{3} /$ $\mathrm{SrTiO}_{3}{ }^{12}$ and $\mathrm{NaNbO}_{3} / \mathrm{SrTiO}_{3}{ }^{13}$ interfaces, likely due to $\mathrm{O}$ vacancies that neutralize hole carries. ${ }^{14,15}$ Indeed, such defects form spontaneously at low $\mathrm{O}$ partial pressure. ${ }^{16}$ At an $n$-type interface, the additional charge from $\mathrm{O}$ vacancies, on the other hand, enhances the electron density. ${ }^{17}$ The quantum gas observed at $\mathrm{LaAlO}_{3} / \mathrm{SrTiO}_{3}$ interfaces, being well confined to the interface region, is commonly attributed to the charge discontinuity at the contact between polar $\mathrm{LaAlO}_{3}$ and non-polar $\mathrm{SrTiO}_{3}$. An increasing thickness of $\mathrm{LaAlO}_{3}$ would result in potential divergence, ${ }^{18}$ the so-called polar catastrophe. Different compensation mechanisms have been proposed ${ }^{14,19,20}$ and are still under debate, ${ }^{21}$ the most famous being electronic reconstruction. Researchers have indicated that the efficiency of electronic reconstruction is supported by multivalent elements, as they enable charge redistribution at lower energy cost. $^{22}$ The ideal number of electrons, that is, 0.5 per perovskite unit cell, as predicted by the polar catastrophe model, could not be confirmed experimentally. ${ }^{21}$ It has been argued that some of the charge may be localized by disorder effects or electron-phonon coupling ${ }^{23}$ and that polarization effects in the presence of the $\mathrm{LaAlO}_{3}$ surface reduce the expected amount of charge. ${ }^{24}$

Because controlling the properties of interphase boundaries requires insight into the mechanisms leading to these properties, in the present work we investigate $\mathrm{SnO} / \mathrm{SnO}_{2}$ (110) interphase boundaries using density functional theory. Although the two component semiconductors $\mathrm{SnO}$ and $\mathrm{SnO}_{2}$ do not give rise to a formal charge discontinuity in the (110) direction, we demonstrate the possibility of forming a hole gas at interphase boundaries (even in the absence of defects) and explain the observations by a generalization of the polar catastrophe model in terms of charge density discontinuity. Charge density discontinuity effects on charge transfer and metallicity at non-stoichiometric oxide interfaces were previously reported in Zhuang et al. ${ }^{25}$ and Jeon et al. ${ }^{26}$ showed that control of the charge transfer makes it possible to stabilize different stoichiometries of molecular ionic salts on metallic substrates.

\section{MATERIALS AND METHODS}

We use the projector augmented wave method with ultrasoft pseudopotentials as implemented in the Vienna Ab initio Simulation Package. ${ }^{27}$ The Perdew-

Physical Science and Engineering Division (PSE), King Abdullah University of Science and Technology (KAUST), Thuwal, Saudi Arabia

${ }^{1}$ Current address: Department of Physics, King Abdulaziz University, Jeddah, Saudi Arabia.

Correspondence: Professor U Schwingenschlögl, Physical Science and Engineering Division (PSE), King Abdullah University of Science and Technology, Thuwal 23955-6900, Saudi Arabia.

E-mail: udo.schwingenschlogl@kaust.edu.sa

Received 24 April 2017; revised 16 November 2017; accepted 20 November 2017 
Burk-Ernzerhof generalized gradient approximation is employed together with a basis consisting of $\mathrm{O} 2 s, 2 p$ and $\mathrm{Sn} 5 s, 5 p$ orbitals and a cutoff energy of $400 \mathrm{eV}$. Wigner-Seitz radii of 1.26 and $1.59 \AA$ are used for $\mathrm{O}$ and $\mathrm{Sn}$, respectively; the Brillouin zone is integrated on a $5 \times 2 \times 1 \Gamma$-centered grid; and a Gaussian smearing of $0.06 \mathrm{eV}$ width is used. The electronic selfconsistency criterion is set to $10^{-5} \mathrm{eV}$, and convergence of the atomic forces is assumed for values $<0.05 \mathrm{eV} / \AA$.

$\mathrm{SnO}_{2}$ has space group $\mathrm{P} 4 / \mathrm{mnm}$ with the lattice parameters $a=b=4.737 \AA$ and $c=3.186 \AA$ and a direct band gap of $3.6 \mathrm{eV} .{ }^{28}$ On the other hand, SnO has space group $P 4 / \mathrm{nmm}$ with the lattice parameters $a=b=3.804 \AA$ and $\mathrm{c}=4.826 \AA^{28}$ and an indirect band gap of $0.7 \mathrm{eV}$ (direct band gap between 2.5 and $3 \mathrm{eV}$ ). ${ }^{29} \mathrm{We}$ obtain band gaps of $0.68 \mathrm{eV}$ (direct) and $0.40 \mathrm{eV}$ (indirect) for the relaxed $\mathrm{SnO}_{2}$ and $\mathrm{SnO}$ unit cells, respectively. The significant underestimation of the band gap of $\mathrm{SnO}_{2}$ is mainly due to the exchange contribution to the exchange-correlation potential, as it can be overcome by hybrid functional calculations. ${ }^{30}$ Although the band gaps are underestimated, we have verified (using the approach of Van de Walle and Martin ${ }^{31}$ and Puthenkovilakam et al. ${ }^{32}$ ) that the type of band alignment at the interface between $\mathrm{SnO}$ and $\mathrm{SnO}_{2}$ agrees with the experiment, ${ }^{33}$ see Figure 1, such that the effects of artifacts of the methodology on the results in the following can be excluded.

As the (110) growth direction is known to be preferred for $\mathrm{SnO}_{2}{ }^{34}$ and has also been reported for $\mathrm{SnO},{ }^{2}$ we built interphase boundaries between the two compounds along this direction using $3 \times 4 \times 3 \quad \mathrm{SnO}_{2}$ and $2 \times 5 \times 3 \mathrm{SnO}$ supercells. This set-up minimizes the lattice mismatch $(3.0 \%$ and $0.1 \%$ along the $a$ and $b$ axes, respectively) and avoids artificial strain effects, giving rise to the two interfaces (I and II). By construction, both interfaces have the composition of the respective bulk material on both sides. The final supercell comprises a computationally challenging number of 672 atoms, for which the positions are relaxed without imposing any restriction.

Although simple cutting would result in polar (110) surfaces for both $\mathrm{SnO}$ and $\mathrm{SnO}_{2}$, there exist energetically favorable nonpolar (110) surfaces. ${ }^{35}$ That of $\mathrm{SnO}_{2}$ is obtained by removing the outermost $\mathrm{O}$ atoms while maintaining the bridging $\mathrm{O}$ atoms, ${ }^{36}$ and that of $\mathrm{SnO}$ is obtained by removing half of the surface atomic species. In principle, therefore, polar-polar, nonpolar-nonpolar and polar-nonpolar (110) interfaces can be formed between $\mathrm{SnO}$ and $\mathrm{SnO}_{2}$. The fully relaxed structures shown in Figure 2 demonstrate that the polar-polar (110) interface is favorable to the nonpolar-nonpolar (110) interface and therefore is studied in the following. This result may be surprising, as both $\mathrm{SnO}$ and $\mathrm{SnO}_{2}$ favor nonpolar (110) surfaces, but is explained by the greater number of dangling bonds at the nonpolar-nonpolar (110) interface. The difference in interface binding energy between the polar-polar and nonpolar-nonpolar (110) interfaces amounts to $1.09 \mathrm{eV}$ per surface unit cell of $\mathrm{SnO}(0.91 \mathrm{eV}$ per surface unit cell of $\mathrm{SnO}_{2}$ ). a

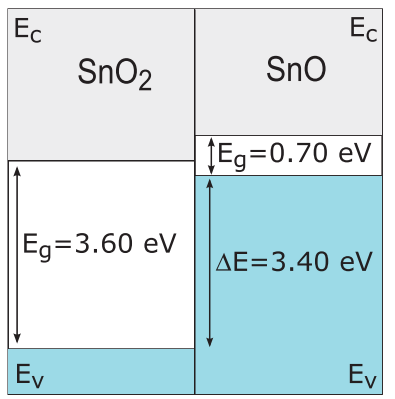

b

\section{Interface I}

\begin{tabular}{|c|c|}
\hline $\mathrm{E}_{\mathrm{c}} \mathrm{SnO}_{2}$ & $\mathrm{SnO}{ }^{E_{c}}$ \\
\hline$\uparrow E_{g}=0.68 \mathrm{eV}$ & $\left\{\begin{array}{l}E_{g}=0.40 \mathrm{eV} \\
\Delta \mathrm{E}=0.18 \mathrm{eV}\end{array}\right.$ \\
\hline$E_{y}$ & \\
\hline
\end{tabular}

Interface II

\begin{tabular}{|l|r|}
\hline$E_{\mathrm{C}} \mathrm{SnO}_{2}$ & SnO $\mathrm{E}_{\mathrm{c}}$ \\
& $\mathrm{E}_{\mathrm{g}}=0.40 \mathrm{eV}$ \\
\hline $\mathrm{E}_{\mathrm{g}}=0.68 \mathrm{eV}$ & $\Delta \mathrm{E}=0.41 \mathrm{eV}$ \\
\hline $\mathrm{E}_{\mathrm{V}}$ & $\mathrm{E}_{\mathrm{V}}$ \\
\hline
\end{tabular}

Figure 1 Band alignment diagrams: (a) Experimental ${ }^{33}$ and (b) theoretical.

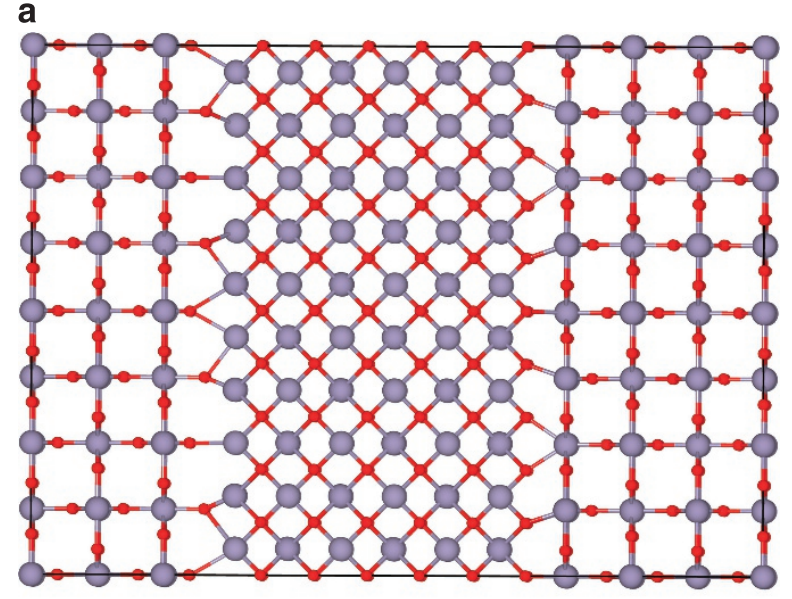

b

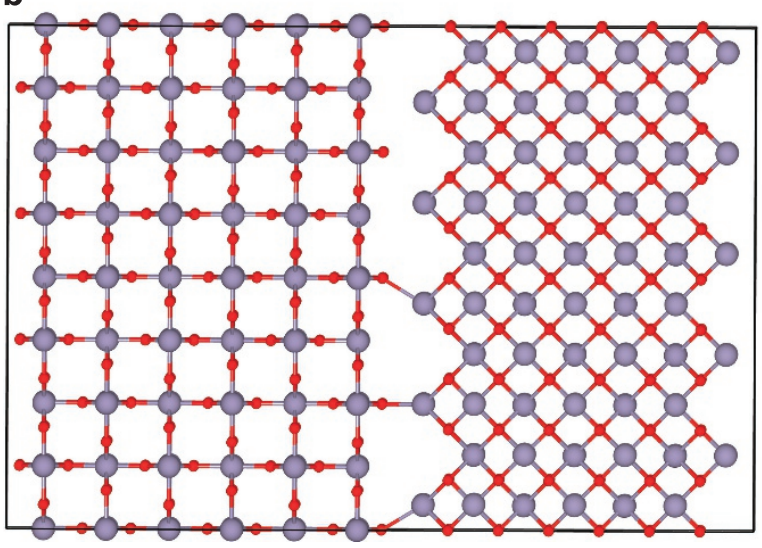

Figure 2 Structurally relaxed (a) polar-polar and (b) nonpolar-nonpolar (110) interfaces.

\section{RESULTS AND DISCUSSION}

Polar catastrophe is usually discussed in terms of formal charge discontinuity rather than charge density discontinuity. However, the density factor becomes important when there is lattice mismatch. We analyze the density of states as a function of the atomic layer number defined in Figure 3a. To this end, partial density of states curves for selected atomic layers are presented in Figure 4 a. Those next to an interface (black lines) show a metallic character, for example, Sn layer 18. We also notice that the $\mathrm{O}$ states of layers 5 and 17 as well as the Sn states of layer 6 shift to higher energy, which indicates that electrons are donated. In contrast, the Sn states of layer 18 shift to lower energy and accept electrons. The atomic layers in the middle of the $\mathrm{SnO}$ and $\mathrm{SnO}_{2}$ slabs still show some metallic states. For thicker slabs, the semiconducting characters of the bulk materials would be fully recovered. However, even if this is not the case for our supercell size, no drawback is expected regarding the validity of our following reasoning because the two interfaces are well separated by almost $15 \AA$.

Structural relaxation effects at interfaces, in general, are strong and therefore have to be taken into account. However, results for the unrelaxed supercell in Figure $4 \mathrm{~b}$ show minor differences with respect to Figure $4 \mathrm{a}$, at least on a qualitative level, suggesting that the physics underlying the creation of metallic states must be of electronic rather than of structural origin. The energetic shifts of the states of the $\mathrm{O}$ terminal layers of $\mathrm{SnO}_{2}$ at interface I (layer 5) and $\mathrm{SnO}$ at interface II (layer 17) are reduced by the structural relaxation, whereas only small modifications affect the two other terminal layers 6 and 18. Minor 


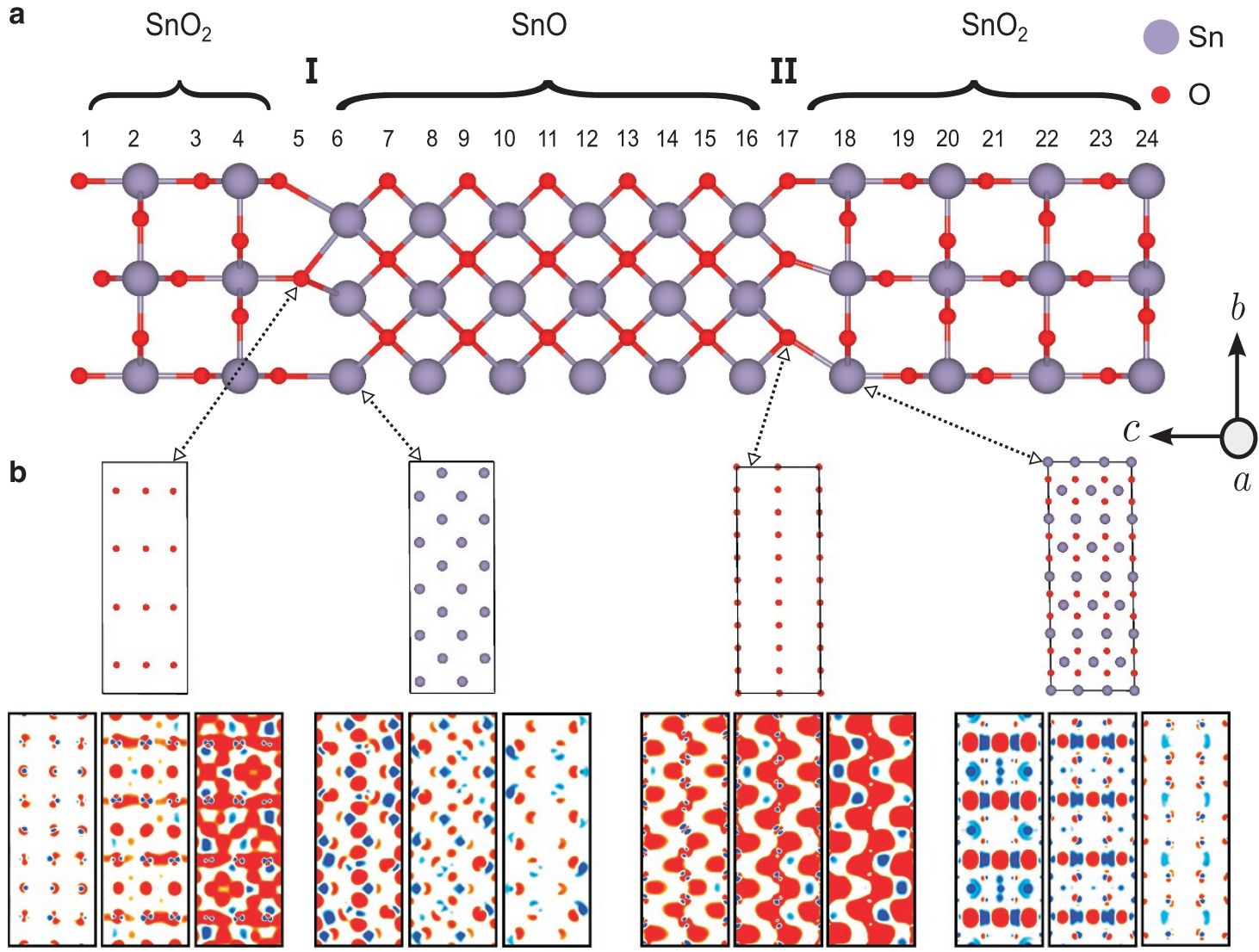

Figure 3 (a) Schematic representation of the $\mathrm{SnO} / \mathrm{SnO}_{2}$ heterostructure with the atomic layers numbered in sequence. (b) Charge density difference maps for the indicated atomic layers and for layers $0.3 \AA$ to the left and to the right. Loss/gain of charge is indicated in red/blue.

effects of the relaxation on the electronic states, as previously reported for the $\mathrm{KTaO}_{3} / \mathrm{SrTiO}_{3}$ interface, are due to small lattice mismatch, ${ }^{37}$ whereas in the present case this is an unexpected finding as the relaxation here is substantial.

To analyze the charge transfer from a spatial perspective, charge density difference maps for the unrelaxed interfaces are depicted in Figure $3 \mathrm{~b}$, with charge loss/gain indicated in red/blue. We show the maps for the highlighted layers as well as for layers $0.3 \AA$ to the left and the right, to track the exact charge transfer scenario. Rather homogeneously distributed holes are observed in the two $\mathrm{O}$ terminal layers 5 and 17. We note that the red circular areas shown in layer 18 are related to the $\mathrm{O}$ atoms in layer 19 , which suggests that the charge transfer is not restricted to the interface itself but reaches one atomic layer farther. Charge differences added for each atomic layer of the relaxed supercell are computed by subtracting the Bader atomic charges from the corresponding charges of the bulk compounds. The results in Figure 5 show the accumulation of 4.9 and 4.5 electrons, respectively, in layers 4 and 18, which reside in Sn $5 p$ orbitals and thus are well localized, supporting our interpretation of Figure $3 \mathrm{~b}$. On the other hand, 3.9, 2.5 and 2.0 holes, respectively, are found in layers 5, 16 and 17 . Furthermore, the $\mathrm{SnO}$ region becomes slightly hole-doped and the $\mathrm{SnO}_{2}$ region slightly electron-doped.

The polarization at polar-polar interfaces can have valence, structural and electronic contributions. ${ }^{38}$ The electronic contribution vanishes in the present case, as both $\mathrm{SnO}$ and $\mathrm{SnO}_{2}$ have a centrosymmetric structure. The structural contribution is given by the rumpling shown in Figure 6. Almost negligible deviations from the bulk value of 0.5 exclude a structural origin of the metallic states. Our next aim is to investigate the valence contribution. Along the (110) direction, $\mathrm{SnO}$ is composed of alternating layers of $\mathrm{O}$ and $\mathrm{Sn}$ atoms, whereas $\mathrm{SnO}_{2}$ consists of alternating layers of $\mathrm{O}$ atoms and $\mathrm{SnO}$ layers. The formal charge thus alternates between +2 and -2 in both cases, and we have no formal charge discontinuity, that is, the electric field oscillates symmetrically around zero such that no potential can build up. Consequently, the polar catastrophe scenario appears not to be relevant in our case. However, we propose a generalized theory: In the absence of formal charge discontinuity, a charge density discontinuity, originating from lattice mismatch, can create a net dipole. This net dipole would lead to potential divergence that must be avoided by means of electronic reconstruction above a critical grain size.

In contrast to interphase boundaries between compounds with the same structure, in our case the formal charge per area is different in the $\mathrm{SnO}$ (110) and $\mathrm{SnO}_{2}$ (110) planes (charge density discontinuity), which we describe in terms of a density factor $G$ assigned to $\mathrm{SnO}$; see Figure 7a. In our supercell, we count at interface I that $24 \mathrm{Sn}^{+2}$ ions meet $20 \mathrm{O}^{-2}$ ions and at interface II that $24 \mathrm{Sn}^{+4}$ and $24 \mathrm{O}^{-2}$ ions meet $20 \mathrm{O}^{-2}$ ions. The density factor consequently amounts to $G=5 / 6$. Figure $7 \mathrm{~b}$ demonstrates (exaggerated for clarity) that the charge density discontinuity leads to an asymmetric oscillation of the electric field around zero and therefore to a potential divergence; see Figure $7 \mathrm{c}$. In the common polar catastrophe model, a valence change in the cations at the interface compensates for the divergence, which has been experimentally confirmed for $\mathrm{LaAlO}_{3} / \mathrm{SrTiO}_{3}{ }^{22}$ and $\mathrm{La}_{0.9} \mathrm{Sr}_{0.1} \mathrm{MnO}_{3} / \mathrm{SrTiO}_{3}{ }^{39}$ However, in principle, different electronic reconstruction scenarios are possible to achieve compensation. The most favorable scenario can be derived from $a b$ initio calculations. 

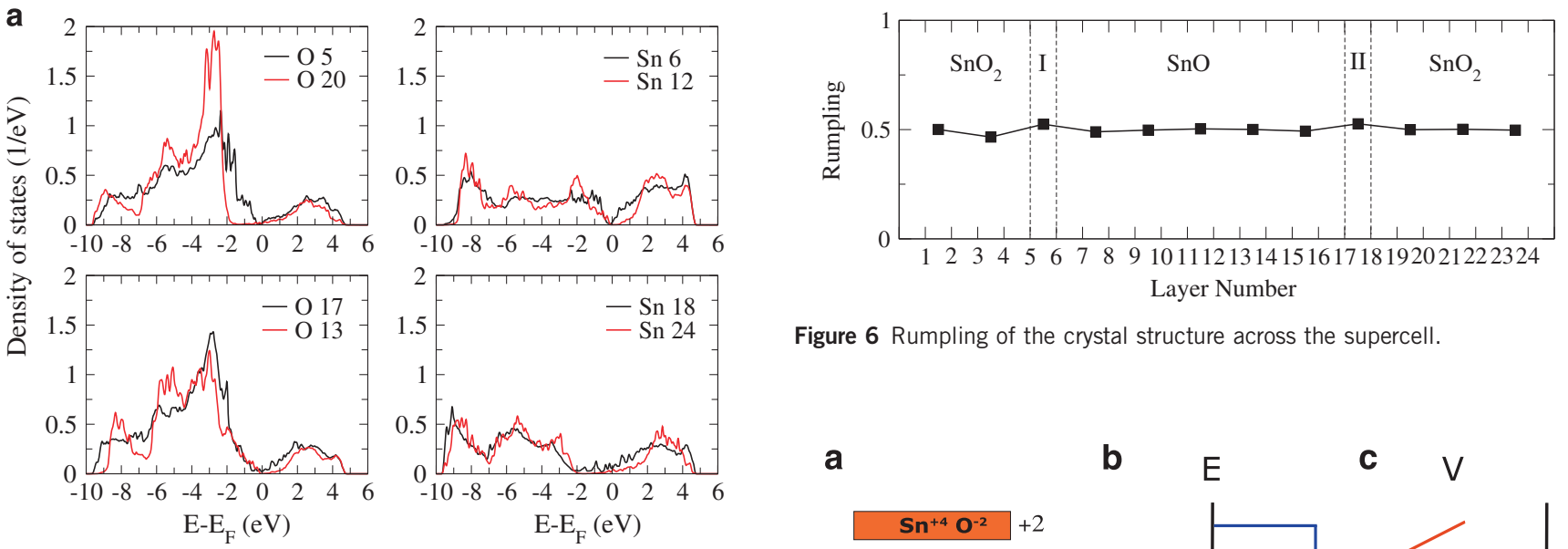

Figure 6 Rumpling of the crystal structure across the supercell.
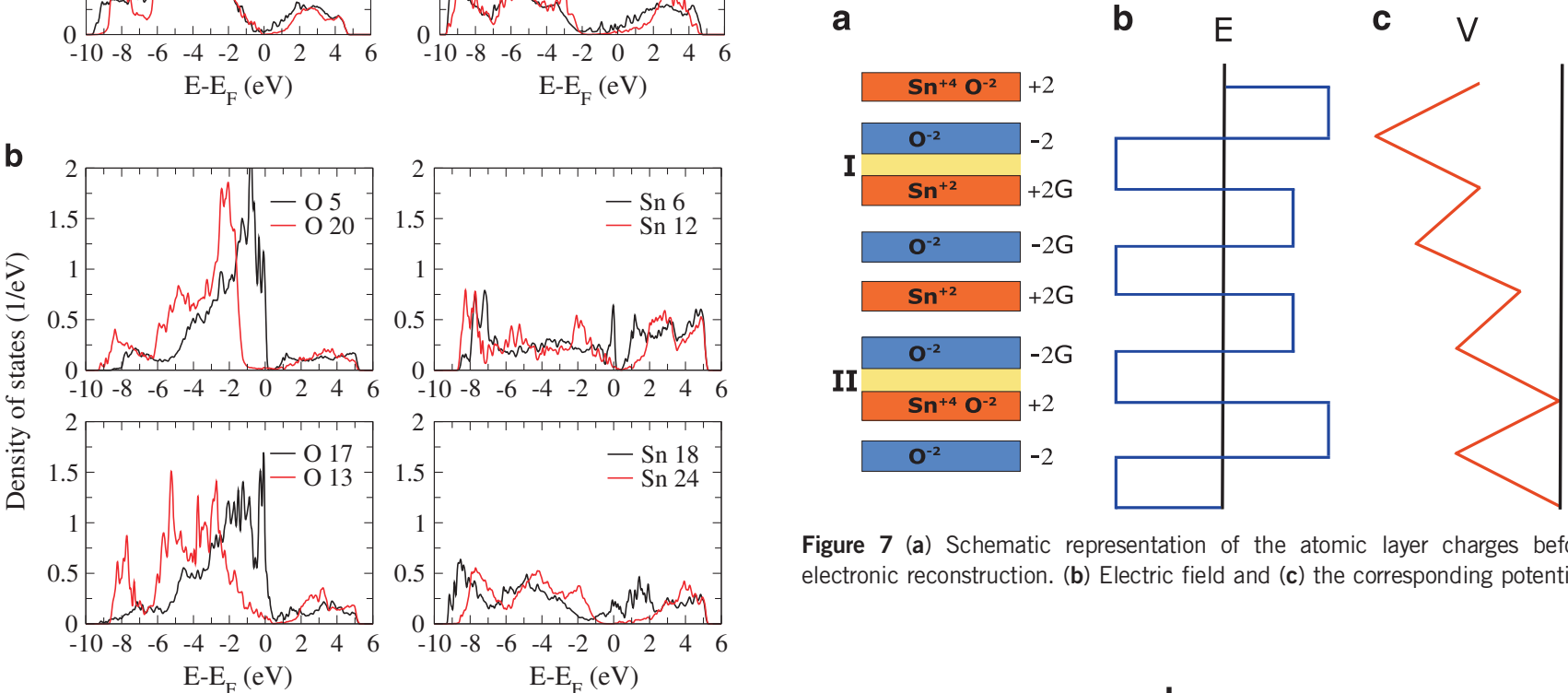

Figure 7 (a) Schematic representation of the atomic layer charges before electronic reconstruction. (b) Electric field and (c) the corresponding potential.

Figure 4 Partial density of states for selected atomic layers in the (a) relaxed and (b) unrelaxed supercells.

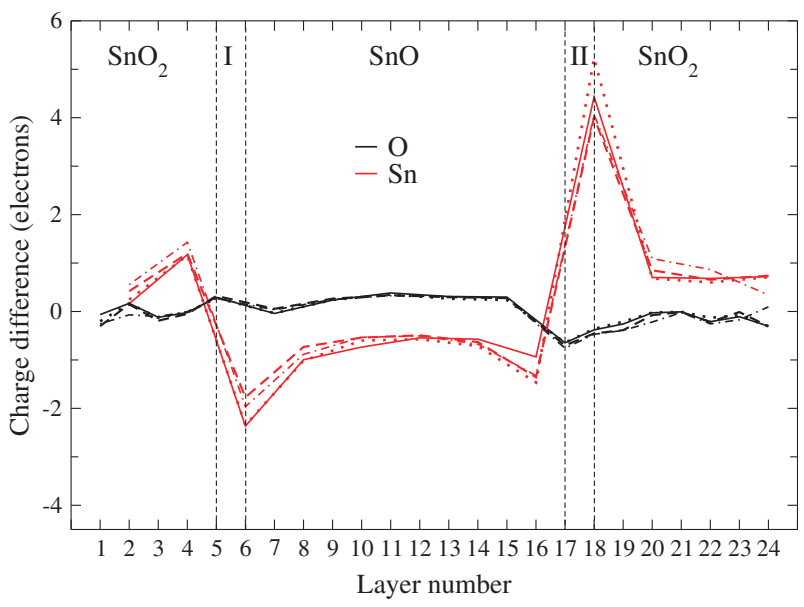

a

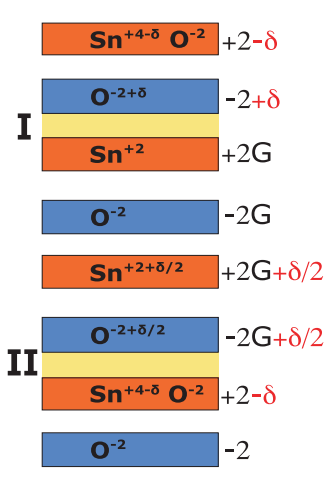

b

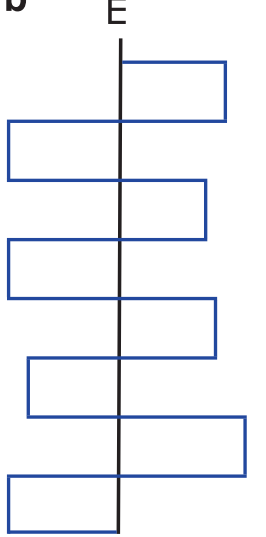

c $\mathrm{V}$

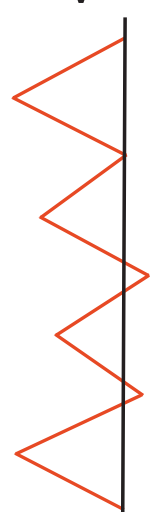

Figure 8 (a) Schematic representation of the atomic layer charges after electronic reconstruction, where $\delta$ is the compensation charge. (b) Electric field and (c) the corresponding potential.

According to Figure 5, at interface I, $\delta$ electrons (polarization discontinuity $^{38}$ ) are accommodated in the Sn $5 p$ orbitals in layer 4 (Sn valence $+4-\delta$ ), and at interface II, $\delta / 2$ holes reside in the Sn $5 p$ orbitals of layer 16 as well as in the O $2 p$ orbitals of layer 17 (Sn valence $+2+\delta / 2$, O valence $-2+\delta / 2$ ). The electronic reconstruction is illustrated schematically in Figure $8 \mathrm{a}$. We note that the generated electric field in this situation, see Figure $8 \mathrm{~b}$, indeed does not give rise to a potential divergence; see Figure $8 \mathrm{c}$. The band alignment diagrams of $\mathrm{SnO}$ and $\mathrm{SnO}_{2}{ }^{33}$ show that the valence band maximum of $\mathrm{SnO}_{2}$ is 


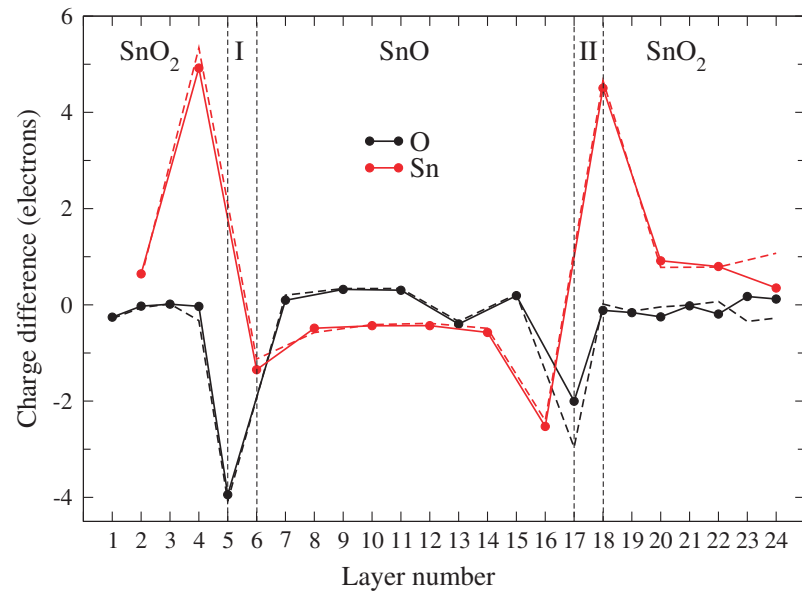

Figure 9 Charge difference per atomic layer in the presence of an 0 vacancy on the $\mathrm{SnO}$ side of interface I (solid line), $\mathrm{SnO}_{2}$ side of interface I (dotted line), $\mathrm{SnO}$ side of interface II (dashed line) and $\mathrm{SnO}_{2}$ side of interface II (dash-dotted line).

approximately $2.5 \mathrm{eV}$ lower than that of $\mathrm{SnO}$. Therefore, charge transfer from $\mathrm{SnO}$ to $\mathrm{SnO}_{2}$ is possible, which fits perfectly with our results, as at interface I the $\mathrm{Sn}$ states on the $\mathrm{SnO}_{2}$ side are occupied by $\delta$ electrons and at interface II the $\mathrm{Sn}$ and $\mathrm{O}$ states on the $\mathrm{SnO}$ side are occupied by $\delta / 2$ holes; see Figure $8 \mathrm{a}$. It is thus the band alignment between the two oxides that results in the specific electronic reconstruction. The formal amount of compensation charge, $\delta=$ $4 / 24=1 / 6$, agrees with the numerical results in Figure 5. Minor deviations can be attributed to partial screening. We note that the proposed mechanism can be generalized directly to other polar-polar interfaces without formal charge discontinuity as long as there is lattice mismatch.

To investigate the effect of $\mathrm{O}$ vacancies on our predictions, we calculate the $\mathrm{O}$ vacancy formation energy for different possible sites with respect to the bulk value and find that $O$ vacancies favor location at the interfaces. We obtain preferences of $0.85 \mathrm{eV}$ for the $\mathrm{SnO}$ side of interface I, $0.85 \mathrm{eV}$ for the $\mathrm{SnO}_{2}$ side of interface I, $1.15 \mathrm{eV}$ for the $\mathrm{SnO}$ side of interface II and $1.32 \mathrm{eV}$ for the $\mathrm{SnO}_{2}$ side of interface II. Figure 9 summarizes results for the charge density difference per atomic layer in the presence of an $\mathrm{O}$ vacancy, showing that there is no qualitative difference in the electronic reconstruction relatively to the case without an $\mathrm{O}$ vacancy.

\section{CONCLUSION}

In conclusion, $a b$ initio calculations reveal the existence of metallic states at (110) interphase boundaries between the semiconductors $\mathrm{SnO}$ and $\mathrm{SnO}_{2}$. This observation is unexpected from the perspective of the common polar catastrophe model that can explain the creation of quantum gases between semiconductors. We therefore propose a generalized model based on charge density discontinuity and demonstrate that it is able to explain electronic reconstruction even at polarpolar interfaces with the same formal charges of the atomic layers on both sides. The introduced mechanism is expected to have an important role at interphase boundaries between polar materials.

\section{CONFLICT OF INTEREST}

The authors declare no conflict of interest.

\section{ACKNOWLEDGEMENTS}

The research reported in this publication was supported by funding from King Abdullah University of Science and Technology (KAUST).

\section{PUBLISHER'S NOTE}

Springer Nature remains neutral with regard to jurisdictional claims in published maps and institutional affiliations.

1 Batzill, M. \& Diebold, U. The surface and materials science of tin oxide. Prog. Surf. Sci. 79, 47-154 (2005).

2 Caraveo-Frescas, J. A., Nayak, P. K., Al-Jawhari, H. A., Granato, D. B., Schwingenschlögl, U. \& Alshareef, H. N. Record mobility in transparent p-type tin monoxide films and devices by phase engineering. ACS Nano 7, 5160-5167 (2013).

3 Shanmugasundaram, A., Basak, P., Satyanarayana, L. \& Manorama, S. V. Hierarchical $\mathrm{SnO} / \mathrm{SnO}_{2}$ nanocomposites: formation of in situ $\mathrm{p}-\mathrm{n}$ junctions and enhanced $\mathrm{H}_{2}$ sensing. Sens. Actuators B 185, 265-273 (2013).

4 Chakhalian, J., Millis, A. J. \& Rondinelli, J. Whither the oxide interface. Nat. Mater. 11 92-94 (2012).

5 Yu, P., Chu, Y. H. \& Ramesh, R. Oxide interfaces: pathways to novel phenomena. Mater Today 15, 320-327 (2012).

6 Ohtomo, A. \& Hwang, H. Y. A high-mobility electron gas at the $\mathrm{LaAlO}_{3} / \mathrm{SrTiO}_{3}$ heterointerface. Nature 427, 423-426 (2004).

7 Zhong, Z., Xu, P. X. \& Kelly, P. J. Polarity-induced oxygen vacancies at $\mathrm{LaAlO}_{3} / \mathrm{SrTiO}_{3}$ interfaces. Phys, Rev. B 82, 165127 (2010).

8 Perna, P., Maccariello, D., Radovic, M., Scotti di Uccio, U., Pallecchi, I., Codda, M. Marré, D., Cantoni, C., Gazquez, J., Varela, M., Pennycook, S. J. \& Granozio, F. M. Conducting interfaces between band insulating oxides: the $\mathrm{LaGaO}_{3} / \mathrm{SrTiO}_{3}$ heterostructure. Appl. Phys. Lett. 97, 152111 (2010).

9 Moetakef, P., Cain, T. A., Ouellette, D. G., Zhang, J. Y., Klenov, D. O., Janotti, A., Van de Walle, C. G., Rajan, S., Allen, S. J. \& Stemmer, S. Electrostatic carrier doping of $\mathrm{GdTiO}_{3} / \mathrm{SrTiO}_{3}$ interfaces. Appl. Phys. Lett. 99, 232116 (2011).

10 Biscaras, J., Bergeal, N., Hurand, S., Grossetête, C., Rastogi, A., Budhani, R. C., LeBoeuf, D., Proust, C. \& Lesueur, J. Two-dimensional superconducting phase in $\mathrm{LaTiO}_{3} / \mathrm{SrTiO}_{3}$ heterostructures induced by high-mobility carrier doping. Phys. Rev. Lett. 108, 247004 (2012)

11 Xu, P., Phelan, D., Jeong, S. J., Mkhoyan, K. A. \& Jalan, B. Stoichiometry-driven metalto-insulator transition in $\mathrm{NdTiO}_{3} / \mathrm{SrTiO}_{3}$ heterostructures. Appl. Phys. Lett. 104, 082109 (2014).

12 Hotta, Y., Susaki, T. \& Hwang, H. Y. Polar discontinuity doping of the $\mathrm{LaVO}_{3} / \mathrm{SrTiO}_{3}$ interface. Phys. Rev. Lett. 99, 236805 (2007).

13 Narkilahti, J., Plekh, M., Levoska, J. \& Tyunina, M. Anomalous growth and properties of $\mathrm{SrTiO}_{3}-\mathrm{NaNbO}_{3}$ superlattices. Phys. Rev. B 79, 014106 (2009).

14 Park, M. S., Rhim, S. H. \& Freeman, A. J. Charge compensation and mixed valency in $\mathrm{LaAlO}_{3} / \mathrm{SrTiO}_{3}$ heterointerfaces studied by the FLAPW method. Phys. Rev. B 74, 205416 (2006).

15 Choi, W. S., Kang, M. G., Do, Y. H., Jung, W. S., Ju, B. K., Yoon, S. J., Yoo, K. S. \& $\mathrm{Kang}, \mathrm{C}$. Y. Oxygen vacancy effects of two-dimensional electron gas in $\mathrm{SrTiO}_{3} / \mathrm{KNbO}_{3}$ hetero structure. J. Sensor Sci. Technol. 22, 244-248 (2013).

16 Zhang, L., Zhou, X. F., Wang, H. T., Xu, J. J., Li, J., Wang, E. G. \& Wei, S. H. Origin of insulating behavior of the p-type $\mathrm{LaAlO}_{3} / \mathrm{SrTiO}_{3}$ interface: polarization-induced asymmetric distribution of oxygen vacancies. Phys. Rev. B 82, 125412 (2010).

17 Siemons, W., Koster, G., Yamamoto, H., Harrison, W. A., Lucovsky, G., Geballe, T. H., Blank, D. H. A. \& Beasley, M. R. Origin of charge density at $\mathrm{LaAlO}_{3}$ on $\mathrm{SrTiO}_{3}$ heterointerfaces: possibility of intrinsic doping. Phys. Rev. Lett. 98, 196802 (2007).

18 Thiel, S., Hammerl, G., Schmehl, A., Schneider, C. W. \& Mannhart, J. Tunable quasi-two-dimensional electron gases in oxide heterostructures. Science 313, 19421945 (2016).

19 Pauli, S. A. \& Willmott, P. R. Conducting interfaces between polar and non-polar insulating perovskites. J. Phys. Condens. Matter 20, 264012 (2008),

20 Bristowe, N. C., Ghosez, P., Littlewood, P. B. \& Artacho, E. The origin of two dimensional electron gases at oxide interfaces: insights from theory. J. Phys. Condens. Matter 26, 143201 (2014).

21 Mannhart, J., Blank, D. H. A., Hwang, H. Y. Millis, A. J. \& Triscone, J. M. Twodimensional electron gases at oxide interfaces. MRS Bull. 33, 1027-1034 (2008).

22 Nakagawa, N., Hwang, H. Y. \& Muller, D. A. Why some interfaces cannot be sharp. Nat. Mater. 5, 204-209 (2006).

23 Popovic, Z. S., Satpathy, S. \& Martin, R. M. Origin of the two-dimensional electron gas carrier density at the $\mathrm{LaAlO}_{3}$ on $\mathrm{SrTiO}_{3}$ interface. Phys. Rev. Lett. 101, 256801 (2008).

24 Behtash, M., Nazir, S., Wang, Y. \& Yang, K. Polarization effects on the interfacial conductivity in $\mathrm{LaAlO}_{3} / \mathrm{SrTiO}_{3}$ heterostructures: a first-principles study. Phys. Chem. Chem. Phys. 18, 6831-6838 (2016).

25 Zhuang, H. L., Zhang, L., Xu, H., Kent, P. R. C., Ganesh, P. \& Cooper, V. R. Tunable one-dimensional electron gas carrier densities at nanostructured oxide interfaces. Sci. Rep. 6, 25452 (2016).

26 Jeon, S., Doak, P. W., Sumpter, B. G., Ganesh, P. \& Maksymovych, P. Thermodynamic control of two-dimensional molecular ionic nanostructures on metal surfaces. ACS Nano 10, 7821-7829 (2016).

27 Kresse, G. \& Joubert, D. From ultrasoft pseudopotentials to the projector augmentedwave method. Phys. Rev. B 59, 1758-1775 (1999). 
28 Cossement, C., Darville, J., Gilles, J., Nagy, J. B., Fernandez, C. \& Amoureux, J. Chemical shift anisotropy and indirect coupling in $\mathrm{SnO}_{2}$ and $\mathrm{SnO}$. Magn. Reson. Chem. 30, 263-270 (1992).

29 Lefebvre, I., Szymanski, M. A., Olivier-Fourcade, J. \& Jumas, J. C. Electronic structure of tin monochalcogenides from SnO to SnTe. Phys. Rev. B 58, 1896-1906 (1998).

30 Behtash, M., Joo, P. H., Nazir, S. \& Yang, K. Electronic structures and formation energies of pentavalent-ion-doped $\mathrm{SnO}_{2}$ : first-principles hybrid functional calculations. J. Appl. Phys. 117, 175101 (2015).

31 Van de Walle, C. G. \& Martin, R. M. Theoretical study of band offsets at semiconductor interfaces. Phys. Rev. B 35, 8154-8165 (1987).

32 Puthenkovilakam, R., Carter, E. A. \& Chang, J. P. First-principles exploration of alternative gate dielectrics: electronic structure of $\mathrm{ZrO}_{2} / \mathrm{Si}$ and $\mathrm{ZrSiO}_{4} / \mathrm{Si}$ interfaces. Phys. Rev. B 69, 155329 (2004).

33 Quackenbush, N. F., Allen, J. P., Scanlon, D. O., Sallis, S., Hewlett, J. A., Nandur, A. S., Chen, B., Smith, K. E., Weiland, C., Fischer, D. A., Woicik, J. C., White, B. E., Watson, G. W. \& Piper, L. F. J. Origin of the bipolar doping behavior of SnO from X-ray spectroscopy and density functional theory. Chem. Mater. 25, 3114-3123 (2013).

34 Duan, Y. Electronic properties and stabilities of bulk and low-index surfaces of SnO in comparison with $\mathrm{SnO}_{2}$ : a first-principles density functional approach with an empirical correction of van der Waals interactions. Phys. Rev. B 77, 045332 (2008).

35 Tasker, P. W. The stability of ionic crystal surfaces. J. Phys. C 12, 4977-4984 (1979).

36 Hong, S.-N., Kye, Y.-H., Yu, C.-J., Jong, U.-G., Ri, G.-C., Choe, C.-S., Kim, K.-H. \& $\mathrm{Han}, \mathrm{J} .-\mathrm{M}$. Ab anitio thermodynamic study of the $\mathrm{SnO}_{2}(110)$ surface in an $\mathrm{O}_{2}$ and $\mathrm{NO}$ environment: a fundamental understanding of the gas sensing mechanism for $\mathrm{NO}$ and $\mathrm{NO}_{2}$. Phys. Chem. Chem. Phys. 18, 31566-31578 (2016).
37 Nazir, S., Singh, N. \& Schwingenschlögl, U. Charge transfer mechanism for the formation of metallic states at the $\mathrm{KTaO}_{3} / \mathrm{SrTiO}_{3}$ interface. Phys. Rev. B 83, 113107 (2011)

38 Noguera, C. \& Goniakowski, J. Oxide materials at the two-dimensional limit. Springer Ser. Mater. Sci. 234, 201-231 (2016).

39 Mundy, J. A., Hikita, Y., Hidaka, T., Yajima, T., Higuchi, T., Hwang, H. Y., Muller, D. A. \& Kourkoutis, L. F. Visualizing the interfacial evolution from charge compensation to metallic screening across the manganite metal-insulator transition. Nat. Commun. 5, 3464 (2014).

This work is licensed under a Creative Commons Attribution 4.0 International License. The images or other third party material in this article are included in the article's Creative Commons license, unless indicated otherwise in the credit line; if the material is not included under the Creative Commons license, users will need to obtain permission from the license holder to reproduce the material. To view a copy of this license, visit http:// creativecommons.org/licenses/by/4.0/

(C) The Author(s) 2018 